\title{
Computation Offloading Decision Bounds in SWIPT-based Fog Networks
}

\author{
Arash Bozorgchenani, Daniele Tarchi, Giovanni Emanuele Corazza \\ Department of Electrical, Electronic and Information Engineering, University of Bologna, Italy \\ Email: \{arash.bozorgchenani2,daniele.tarchi,giovanni.corazza\}@unibo.it
}

\begin{abstract}
Computation sharing is one of the most promising services in fog computing allowing the Fog Nodes (FNs) to share among themselves data and tasks to be computed. In case of battery powered-FNs, energy consumption becomes an issue. Simultaneous Wireless Information and Power Transfer (SWIPT) is a recently introduced technology enabling data and power transfer through microwave links among different nodes. In this work, we have considered the presence of a battery powered FN able to simultaneously share data and harvest energy from a Fog Access Point (F-AP), supposed to be plugged to the electrical network. The aim of this work is to define two suitable bounds able to drive the offloading decision to be taken by the battery powered FN, based on the estimated packet generation time, with the aim of having a stable energy system. We have further studied the impact of bandwidth and packet size on the two bounds. Simulation results demonstrate the impact of SWIPTbased offloading decision algorithm on network in terms of latency and network lifetime.
\end{abstract}

\section{INTRODUCTION}

Many mobile applications run on devices with limited resources. These applications use the wireless networks and consume some resources, e.g., processor, battery, storage. In an Internet of Things (IoT) scenario, with huge amount of devices with limited resources and capabilities, there is a large demand for resources that can not be responded by the devices themselves. Fog computing is a technology enabling the presence of more powerful devices, called FogAccess Points (F-APs), to assist the edge devices in their demands by sharing their resources. Fog networking, initiated by Cisco, enables the fog computing technology at the network edge. Fog networking mainly features ubiquity, distributed management and cooperation among the edge devices [1].

Computation offloading is one of the possible services that can be deployed through a fog network, aiming at migrating the computation from the hungry edge devices, Fog Nodes (FNs), to the resource-rich devices, F-APs. However, when offloading, there is an extra transmission and reception time for which the FN also consumes a certain amount of energy. As a result, computation offloading in battery powered devices involves a trade-off between energy consumption and processing delay, impacting the offloading decision.

This work has been partially supported by the project "GAUChO - A Green Adaptive Fog Computing and Networking Architecture" funded by the MIUR Progetti di Ricerca di Rilevante Interesse Nazionale (PRIN) Bando 2015 grant 2015YPXH4W_004.
This trade-off can be addressed by the exploitation of microwave links for transferring also energy apart from the information. Wireless Power Transfer (WPT) technology is a promising candidate for energy related issues in a wireless network. Several WPT applicability experiments were conducted in its early stage; among them, it is worth to mention a wireless powered helicopter, flying at 60 feet above the ground level by William C.Brown in 1963 [2]. In 2008, the idea of simultaneously transmit power and data through a wireless link was firstly proposed at MIT [3]. In microwave WPT systems, Direct Current (DC) power can be converted to Radio Frequency (RF) power using an amplifier, while at the receiver side the RF power can be converted back to the DC power using a rectifier [4]. As a result, a wireless interaction between the F-AP and the FN can be done not only for sharing computational resources, but also for sharing energy. This wireless charging technique is called Simultaneous Wireless Information and Power Transfer (SWIPT) which is expected to extend the battery life time [5].

In SWIPT the trade-off between energy consumption and task offloading latency is moved from the devices to the links requesting a different approach in designing the system. This is particularly important in a Fog Computing scenario that is characterized by heterogeneous applications having different characteristics in terms of task sizes, processing load, link bandwidth. In this work, we exploit the SWIPT technology in a fog computing scenario. The FN can offload its task to the energy beacon enabled F-APs. The FN can harvest energy from the F-AP when the communication channel is idle. We have analyzed the computation offloading under different bandwidth and packet sizes in order to find the bounds allowing the FN to make an offloading decision such that the system is stable from the energy point of view.

\section{System Model and Problem Formulation}

The system is composed by two types of edge nodes, named F-APs and FNs. Both edge nodes (either the FN or the FAP) have computational and storage capabilities. However, the F-AP is supposed to have higher computational capabilities than the FN. On the other side, the FN can either perform the computation of a given task locally, or offload it to the nearby F-AP. In particular we are focusing on a single link between one FN and one F-AP, interacting for offloading the 
computational effort and harvesting energy through the SWIPT technology.

We have assumed that a power beacon is integrated with the F-AP so that the deployed power beacon can radiate power to the FN. Thus, the FN is able to harvest some amount of energy from the F-AP by using the SWIPT technology. We have considered a time division approach between wireless and power transfer so that the power can be transferred only during communication idle periods [6].

The focus of this work is to define appropriate bounds for the offloading decision with the aim of remaining in an energy stability region by properly exploiting the SWIPT technology. To this aim, when an FN has a task to be processed, it can either compute it locally or offload the processing to the F-AP, as long as the FN is within its coverage area.

The overall energy consumed by the FN up to the time instant $t$ is:

$$
E_{c}^{F N}(t)=P_{t x}^{F N} t_{t x}^{F N}+P_{r x}^{F N} t_{r x}^{F N}+P_{c o m}^{F N} t_{c o m}^{F N}+P_{i d}^{F N} t_{i d}^{F N}
$$

where $P_{t x}^{F N}, P_{r x}^{F N}, P_{c o m}^{F N}$ and $P_{i d}^{F N}$ are, respectively, the power consumption when in transmission, reception, computation and idle, while, $t_{t x}^{F N}, t_{r x}^{F N}, t_{c o m}^{F N}$ and $t_{i d}^{F N}$ are, respectively, the amount of time the $\mathrm{FN}$ is in transmission, reception, computation and idle up to the time $t$. It is worth to be noticed that the communication circuitry is separated from the computational circuitry, hence, an FN can transmit/receive while computing; this means that in general:

$$
t \leq t_{t x}^{F N}+t_{r x}^{F N}+t_{c o m}^{F N}+t_{i d}^{F N}
$$

where the equality occurs if and only if the transmit/receive phase and the computing phase are completely disjoint. Otherwise, the sum of the four terms is higher than the considered interval.

By supposing to use the SWIPT technology between the F-AP and the FN with the time division approach, we can consider that the FN can harvest energy [5]. We define the received power at the $\mathrm{FN}$ as [2]:

$$
P_{h}^{F N}=\eta^{h} P_{t x}^{F-A P}|h|^{2}
$$

where $\eta^{h}$ is the power transfer efficiency, $|h|^{2}$ is the channel gain between the FN and the F-AP and $P_{t x}^{F-A P}$ is the power transmitted by the F-AP to be harvested by the FN.

We assume that the FN can harvest energy when its communication circuitry is not used (i.e., when it is neither transmitting nor receiving). Thus, at a certain time instant $t$, the overall harvested energy can be defined as:

$$
E_{h}^{F N}(t)=P_{h}^{F N} \cdot\left(t-t_{t x}^{F N}-t_{r x}^{F N}\right)
$$

If we suppose that the initial energy of the $\mathrm{FN}$ is $E_{r}^{F N}(0)$, the remained energy of the $\mathrm{FN}$ at certain time instant $t$, considering the harvested and consumed energy can be calculated as:

$$
E_{r}^{F N}(t)=E_{r}^{F N}(0)-E_{c}^{F N}(t)+E_{h}^{F N}(t) .
$$

In the considered fog computing scenario, the $\mathrm{FN}$ consumes some amount of energy during each of the states defined in (1), and harvests some amounts while the antenna is free. Thus, in order to have the system in a stable state from the energy point of view, the following should hold:

$$
E_{h}^{F N}(t) \geq E_{c}^{F N}(t)
$$

which means the total harvested energy up to the time $t$ by the FN should be greater than the total consumed power. If the above condition is true, the network is alive at least up to time instant $t$.

Let us focus on a time interval $T$ between two task generations at the $\mathrm{FN}$ to be computed, by considering that the packet generation rate follows a Poisson distribution with average $\lambda$ generated packets per second. Let us define the time for locally computing at the $\mathrm{FN}$ a task as $T_{\text {com }}=O / \eta_{\mathrm{com}}^{F N}$, where $O$ and $\eta_{c o m}^{F N}$ are the number of operations to process a task and the computational power of the FN, respectively. On the other side, in case of offloading, we define $T_{t x}=L_{d} / r$ and $T_{r x}=L_{r} / r$ as the transmission and reception time interval for a single task, where $L_{d}, L_{r}$ and $r$ are the transmitted packet size for the related task generated at the FN, the packet size of the result of the offloaded processing, and the data rate of the link between the FN and the F-AP, respectively. Now, by considering (1) and (3), if we focus on a single inter-arrival time interval $T$ we can rewrite (5) as:

$$
\begin{aligned}
P_{h}^{F N} & \left(T-\alpha \frac{L_{d}}{r}-\alpha \frac{L_{r}}{r}\right) \geq \\
& P_{i d}^{F N}\left(T-\alpha \frac{L_{d}}{r}-\alpha \frac{L_{r}}{r}-(1-\alpha) \frac{O}{\eta_{c o m}^{F N}}\right) \\
& +\alpha P_{t x}^{F N} \frac{L_{d}}{r}+\alpha P_{r x}^{F N} \frac{L_{r}}{r}+(1-\alpha) P_{c o m}^{F N} \frac{O}{\eta_{c o m}^{F N}}
\end{aligned}
$$

where $\alpha$ equals to 1 if the FN is offloading to the F-AP and 0 if it is performing a local computation.

Now, we are interested in finding the minimum packet interarrival time to harvest enough energy in order to respect (5). We define $T_{l o c}$ as the packet inter-arrival time when the FN performs a local computation (i.e., $\alpha=0$ ), and rewrite (6) as:

$$
P_{h}^{F N} \cdot T_{l o c} \geq P_{i d}^{F N}\left(T_{l o c}-\frac{O}{\eta_{c o m}^{F N}}\right)+P_{c o m}^{F N} \frac{O}{\eta_{c o m}^{F N}}
$$

Through simple algebraic operations, it is possible to set a lower bound for having respected the energy stability condition in (5) in case of local processing, as:

$$
T_{l o c} \geq \frac{\frac{O}{\eta_{c o m}^{F N}}\left(P_{c o m}^{F N}-P_{i d}^{F N}\right)}{P_{h}^{F N}-P_{i d}^{F N}}
$$

The obtained bound can be seen as a threshold for understanding if the packet generation inter-arrival time allows to remain in the energy stability condition when performing a local processing. In case the inter-arrival time is higher we have sufficient amount of energy that is harvested, otherwise it is not possible to harvest sufficient amount of energy for processing that packet locally.

On the other hand, if we consider the case in which the FN offloads the computation (i.e., $\alpha=1$ ), it is possible to 
set a lower bound to the time interval for having respected the energy stability condition. We define $T_{\text {off }}$ as the packet inter-arrival time when the FN offloads the packet; hence, (6) can be written as:

$$
\begin{aligned}
& P_{h}^{F N}\left(T_{o f f}-\frac{L_{d}}{r}-\frac{L_{r}}{r}\right) \geq \\
& P_{i d}^{F N}\left(T_{o f f}-\frac{L_{d}}{r}-\frac{L_{r}}{r}\right)+P_{t x}^{F N} \frac{L_{d}}{r}+P_{r x}^{F N} \frac{L_{r}}{r}
\end{aligned}
$$

Through simple algebraic operations, it is possible to set a lower bound for having respected the energy stability condition in (5) in case of offloaded processing, as:

$$
\begin{aligned}
& T_{o f f} \geq \\
& \frac{\frac{L_{d}}{r}\left(P_{t x}^{F N}+P_{h}^{F N}-P_{i d}^{F N}\right)+\frac{L_{r}}{r}\left(P_{r x}^{F N}+P_{h}^{F N}-P_{i d}^{F N}\right)}{P_{h}^{F N}-P_{i d}^{F N}}
\end{aligned}
$$

The obtained bound can be seen as a threshold for understanding if the packet generation inter-arrival time allows to remain in the energy stability condition when offloading the process. In case the inter-arrival time is higher we have sufficient amount of energy that is harvested, otherwise it is not possible to harvest sufficient amount of energy for offloading the computation.

The obtained bounds give two thresholds that can be exploited for deciding whether offloading or not in order to remain in energy stability.

\section{OfFlOADING DECISION-MAKING APPROACH}

Following the bounds definition in (8) and (10), it is possible to relate them with the packet interarrival time for estimating the decision to be taken. Hence, we make an estimation of the arrival time of the next packet and, based on the estimated time, we can decide whether the FN can perform a local computation or offload in order to respect the condition in (5).

We consider that the arrival time of the packets follows a Poisson distribution with average arrival $\lambda$ packets per second. We define $\bar{T}^{l}(t)$ as the estimated inter-arrival time of the $l$ th packet at time instant $t$, as:

$$
\bar{T}^{l}(t)=\sum_{i=1}^{N} \alpha_{i} T_{A}^{l-i}
$$

where $T_{A}^{l-i}$ indicates the measured inter-arrival time of the $(l-$ $i)$ th packet, and $\alpha_{i}$ is an opportunely set parameter allowing to consider a window of $N$ previously measured inter-arrival intervals.

The offloading decision is based on comparing the estimated inter-arrival time with the two thresholds in (8) and (10), i.e.,

$$
\left\{\begin{array}{l}
\bar{T}^{l}(t) \gtrless T_{l o c} \\
\bar{T}^{l}(t) \gtrless T_{\text {off }} .
\end{array}\right.
$$

To this aim it is worth to be noticed that the greater between $T_{l o c}$ and $T_{\text {off }}$ depends on several parameters, among which bandwidth and the packet length, while considering fixed the

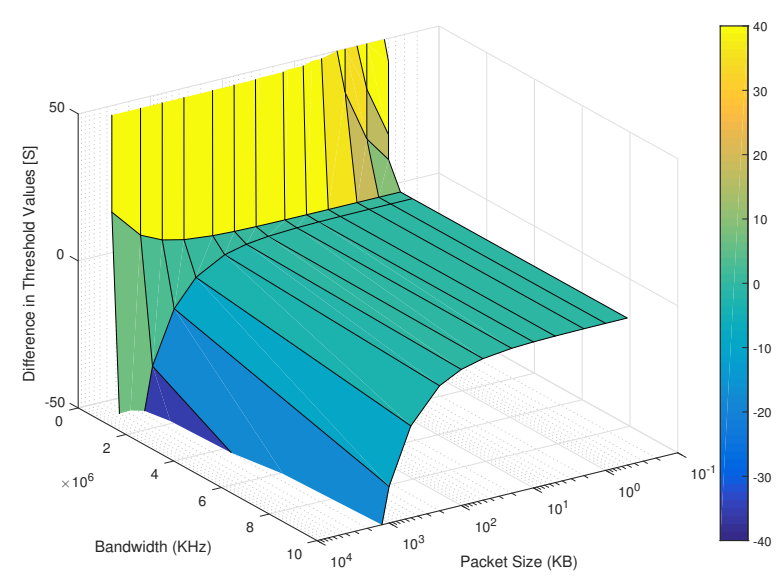

Fig. 1. Difference between the offloading and local thresholds $\left(T_{o f f}-T_{l o c}\right)$

power related terms. By analyzing both (8) and (10), it is possible to notice that higher is the bandwidth, the lower is the interaction time, favoring to offload the tasks. However, if the bandwidth is smaller due to the longer communication time, a local computation might be better in terms of time. On the other hand, the length of a packet impacts on the computation, reception and transmission times. To this aim, this motivates us to analyze the impact of these two parameters on the two thresholds.

We have considered the difference between the offloading and local thresholds $\left(T_{o f f}-T_{l o c}\right)$ by considering variable bandwidth and packet sizes. The result is depicted in Fig. 1 and Tab. I.

As seen in Tab. I, when the bandwidth is low and packet size is small, the offloading threshold is slightly greater than local threshold; however, as the packet size increases, the offloading threshold gets much larger than the local threshold. This means in low bandwidth and large packet size performing a local computation is more beneficial. On the other hand, when the bandwidth is high, with small packet size local threshold is slightly greater than the offloading threshold, however, when the packet size increases, this difference also rises. This indicates that with high bandwidth and different packet sizes, offloading is desirable because it takes a shorter time to harvest sufficient amount of energy to make up for the consumed energy required for the processing the task.

The previous analysis allows to individuate three areas. Area 1 represents the inter-arrival times lower than the lowest threshold. Area 2 is the intermediate area, representing the inter-arrival times where it is possible to make an offloading decision allowing to harvest sufficient amount of energy, while in Area 3 both offloading decisions allow to harvest a sufficient amount of energy.

The offloading decision, hence, leads to two cases depending on the order of the thresholds, represented in Fig. 2. If the estimated arrival time is lower than the lowest of the two 
TABLE I

THRESHOLD DIFFERENCES IN SECONDS WITH DIFFERENT BANDWIDTH AND PACKET SIZE.

\begin{tabular}{r|rrrrrrrrrrrrr}
\hline & $500 \mathrm{~B}$ & $1 \mathrm{kB}$ & $2 \mathrm{kB}$ & $5 \mathrm{kB}$ & $10 \mathrm{kB}$ & $20 \mathrm{kB}$ & $50 \mathrm{kB}$ & $100 \mathrm{kB}$ & $200 \mathrm{kB}$ & $500 \mathrm{kB}$ & $1 \mathrm{MB}$ & $2 \mathrm{MB}$ & $5 \mathrm{MB}$ \\
\hline $200 \mathrm{~Hz}$ & 42 & 84 & 168 & 420 & 840 & 1680 & 4200 & 8401 & 16803 & 42007 & 84015 & 168029 & 420074 \\
$500 \mathrm{~Hz}$ & 17 & 34 & 68 & 172 & 344 & 689 & 1724 & 3449 & 6898 & 17247 & 34494 & 68988 & 172469 \\
$1 \mathrm{kHz}$ & 8 & 17 & 35 & 87 & 175 & 351 & 879 & 1758 & 3517 & 8794 & 17589 & 35177 & 87943 \\
$500 \mathrm{kHz}$ & 0.001 & 0.003 & 0.006 & 0.017 & 0.034 & 0.068 & 0.17 & 0.34 & 0.68 & 1.7 & 3.4 & 6.8 & 17 \\
$1 \mathrm{MHz}$ & -0.008 & -0.017 & -0.035 & -0.088 & -0.177 & -0.354 & -0.886 & -1.7 & -3.5 & -8.8 & -17 & -35 & -88 \\
$10 \mathrm{MHz}$ & -0.018 & -0.037 & -0.075 & -0.187 & -0.375 & -0.751 & -1.87 & -3.75 & -7.51 & -18 & -37 & -75 & -187 \\
\hline
\end{tabular}

A)

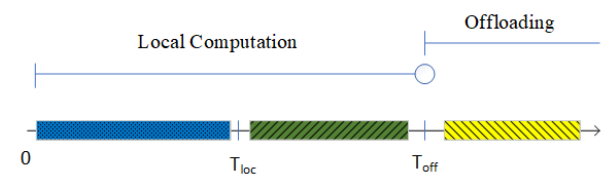

B)

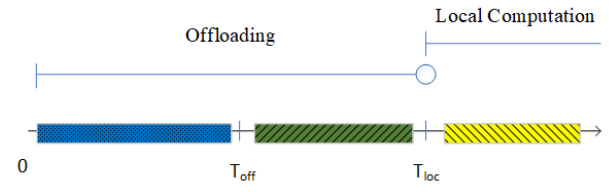

The area the

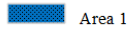

WIDIA Area estimated time lies in

Fig. 2. The offloading and local computation thresholds.

thresholds the FN cannot harvest sufficiently respecting (5), and it is supposed to make the decision based on the lower threshold, meaning local computation in case (A), and offloading in case (B). On the other hand, if the estimated arrival time is greater than the lowest threshold, the FN is able to harvest sufficiently if it makes the decision only based on the lowest threshold; meaning local computation in case (A), and offloading in case (B). However, if the estimated interarrival time is greater than both thresholds the FN can harvest sufficiently regardless of the decision it makes, either offload or not. In this case, we further improve the decision by opting the solution resulting in minimizing the task processing time. To this aim we define the task processing time, when the FN performs a local computation, as:

$$
D_{l o c}^{l}=\frac{O}{\eta_{c o m}^{F N}}
$$

while if the FN offloads the computation to the F-AP, the task delay is:

$$
D_{o f f}^{l}=\frac{L_{d}}{r}+\frac{L_{r}}{r}+\frac{O}{\eta_{c o m}^{F-A P}}
$$

The overall decision Algorithm 1 can be summarized in this way. Let us focus on a task $\Delta^{l}$ transmitted by the considered FN; the offloading process can be identified by a tuple $\left\langle L_{d}, L_{r}, O, r\right\rangle_{l}$. Following the parameters of the $l$ th task it is possible to calculate the related local and offloading thresholds $T_{l o c}^{l}$ and $T_{o f f}^{l}$, following (8) and (10). The comparison between the two thresholds leads to one of the two cases represented in Fig. 2; the decision $\zeta_{l}$ can be performed by comparing the estimated interarrival time with the obtained thresholds.

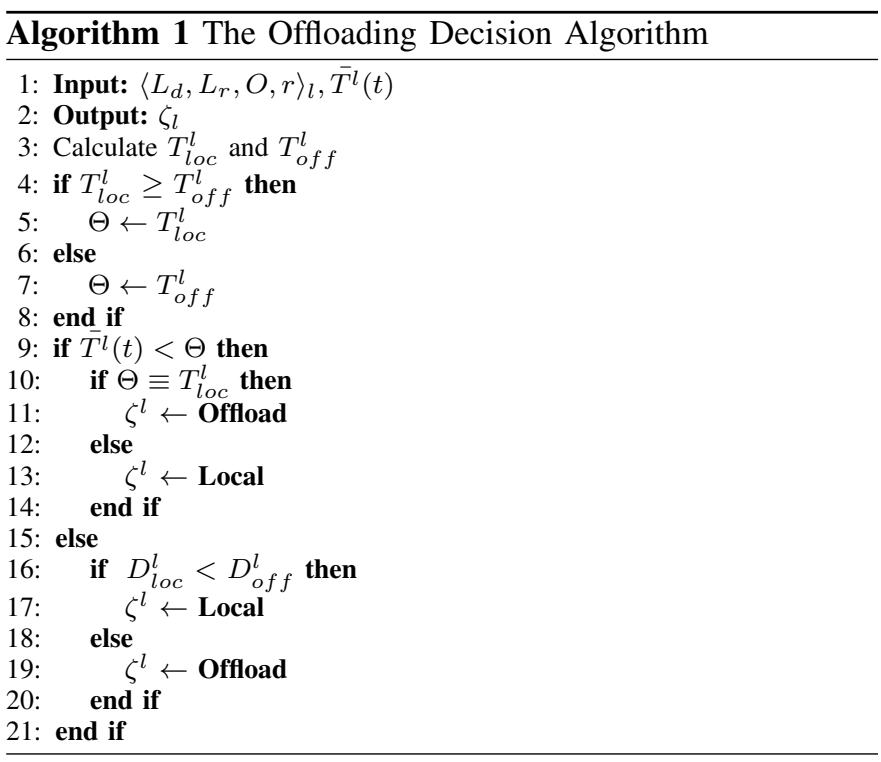

The algorithm first finds the upper threshold (Lines 4-8) and then the decision is made based on the lower threshold (Lines 10-14). When the estimated arrival time of next packet is greater than both thresholds, the offloading decision is made by considering the action resulting in a lower delay (Lines 1620).

\section{NumericAl RESUlts}

In this section, the numerical results obtained through computer simulations in Matlab are presented, where the considered parameters are listed in Tab. II. Each simulation is supposed to run for $3000 \mathrm{~s}$ and the initial energy of the FN, $E_{r}^{F N}(0)$, is considered to be $20 \mathrm{~J}$.

By resorting to [2], in an urban environment, the power transfer efficiency $\eta^{h}$ can be on the order of $1 \%$, when the distance between the beacon and the $\mathrm{FN}$ is on the order of 10 meters; to this aim we set $\eta^{h}=1 \%$ and the FN is $10 \mathrm{~m}$ away from the power beacon.

Remark 1: (Safety Levels of Human Exposure to RF Electromagnetic Fields): According to the IEEE Standard C95.12005 , for safety levels with respect to human exposure to RF electromagnetic fields, the permissible exposure level from $2 \mathrm{GHz}$ to $100 \mathrm{GHz}$ in a public environment is $10 \mathrm{~W} \mathrm{~m}^{-2}[7$, p. 27]. Due to the fact that typical frequencies that are used 
TABLE II

SiMULATION PARAMETERS

\begin{tabular}{|l|l|}
\hline Parameter & Value \\
\hline \hline Task result size $\left(L_{r}\right)$ & $L_{d} / 5$ \\
\hline Path loss exponent & 2.7 (urban area) \\
\hline F-AP coverage range & $50 \mathrm{~m}$ \\
\hline Task Operations $(O)$ & 10000 FLOPs per Byte \\
\hline FN Processing Speed $\left(\eta_{c o m}^{F N}\right)$ & 15 GFLOPS \\
\hline F-AP Processing Speed $\left(\eta_{\text {com }}^{F-A P}\right)$ & 150 GFLOPS \\
\hline FN Computation power $\left(P_{c o m}^{F N}\right)$ & $0.9 \mathrm{~W}$ \\
\hline FN Idle power $\left(P_{i d}^{F N}\right)$ & $0.01 \mathrm{~W}$ \\
\hline $\begin{array}{l}\text { FN Transmission and reception power } \\
\left(P_{t x}^{F N}, P_{r x}^{F N}\right)\end{array}$ & $1.3 \mathrm{~W}$ \\
\hline
\end{tabular}

for far-field WPT systems development are $2.45 \mathrm{GHz}$ and $5.8 \mathrm{GHz}$, we need to define the minimum distance between the receiver and the beacon power in order to respect the standard. If effective radiated power at the power beacon is $\left(P_{\text {erp }}\right)$ and Euclidean distance between the power beacon and receiver is $d$ and considering the power density formula the minimum distance between the two nodes respecting the mentioned permissible exposure level, is defined as:

$$
P_{\text {erp }} /\left(4 \pi d^{2}\right)<10
$$

In this work we have set the radiated power to $1.5 \mathrm{~W}$; hence, the minimum distance in meters is:

$$
d>\sqrt{1.5 / 40 \pi} \simeq 0.1092
$$

Therefore, the radius of exclusion zone is set to this minimum distance considering a far-field WPT.

We have performed the simulation results for variable bandwidth, packet length and $\lambda$, while the estimation of the interarrival time has been performed by considering a constant averaging window over the past $N=5$ packets (i.e, $\alpha_{i}=0.2$ )=. In the following, we will be briefly studying the results for each parameter.

In Fig. 3 the impact of bandwidth is analyzed for different bandwidth values. The packet size is fixed to $1 \mathrm{kB}$ and $\lambda$ is set to 5.5 packets per second. As seen in the figure, as the bandwidth increases the estimated arrival time of the next packet is larger than both thresholds and it falls in the 3rd area. That is due to the fact that by having higher bandwidth, the transmission and reception time decrease as well and the overall task delay gets smaller so that the FN can harvest in a shorter time the consumed energy. Therefore, by the arrival of next packet, the FN has harvested the consumed energy with a high probability (i.e., about $80 \%$ ) when the bandwidth is higher than or equal to $1 \mathrm{MHz}$. However, when the bandwidth is smaller, due to the longer interaction time, a higher delay is experienced and the arrival time usually falls in the second and first area. This result is in accordance with Fig. 2.

To see the impact of the packet size on the two thresholds, numerical results for variable packet lengths have been considered by fixing the bandwidth to $500 \mathrm{kHz}$ and $\lambda$ to 5.5 packets per second. We have selected $500 \mathrm{kHz}$ for the bandwidth, because it allows to have the inter-arrival time falling in all

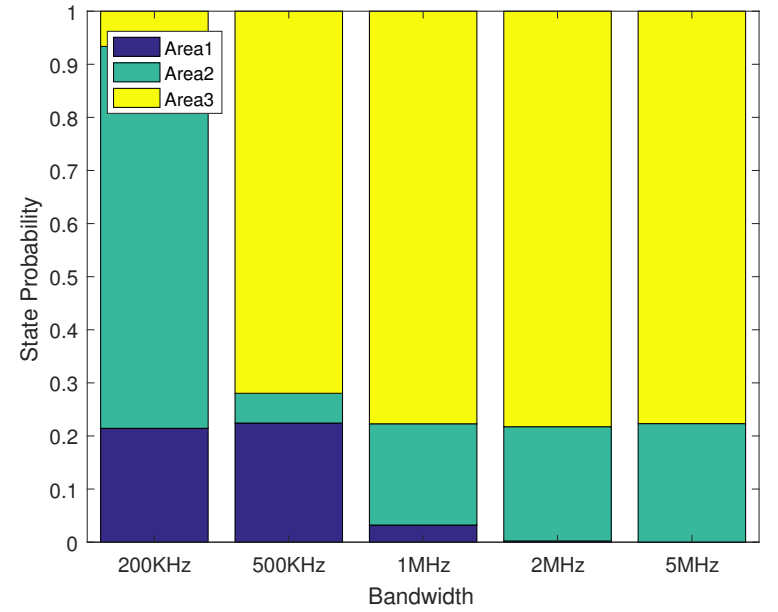

Fig. 3. Threshold Areas for different bandwidth

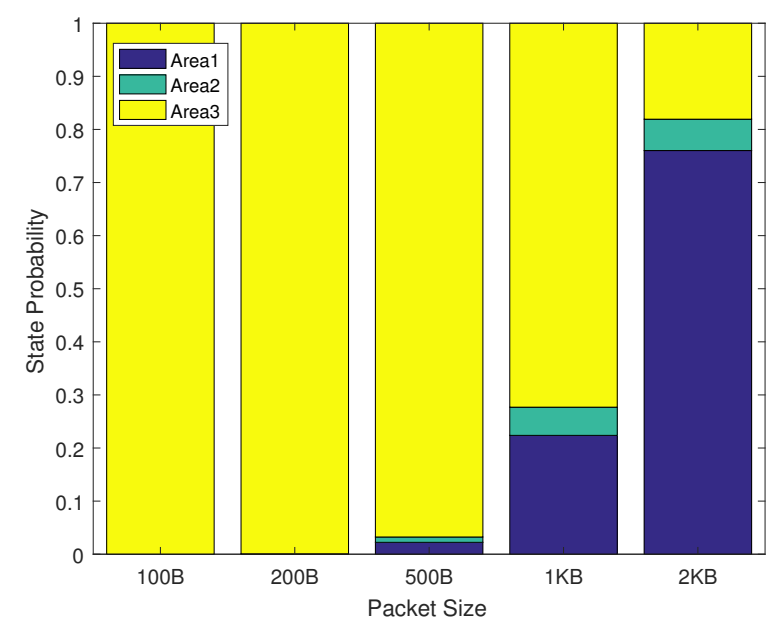

Fig. 4. Threshold Areas for different packet size

areas based on Fig. 3. As seen in Fig. 4, when the packet size is smaller the processing time is smaller as well and there is sufficient time for the FN to harvest; however, when the packet size is large it takes a longer time to process the task resulting in a shorter time for harvesting. This is why the arrival of next packet is earlier than the time required for harvesting and the next packet inter-arrival time mostly lies in area 1 and 2. The other consideration is that due to the value of the bandwidth, i.e, $500 \mathrm{kHz}$, the difference between the two thresholds is reduced; this is in line with Fig. 2 and Tab. I.

Finally, the impact of variable $\lambda$ on the network delay and lifetime is investigated. We have considered a bandwidth equal to $500 \mathrm{kHz}$ and a packet size equal to $1 \mathrm{kB}$ allowing to have the arrival time in the all areas according to Figs. 3 and 4.

The comparison is performed by considering the proposed decision algorithm, where the packet inter-arrival time is estimated and compared with the decision thresholds. The proposed algorithm, labeled SWIPT \& Opt.Thre, is compared with 4 benchmarks: SWIPT \& Loc and SWIPT \& Off consider 


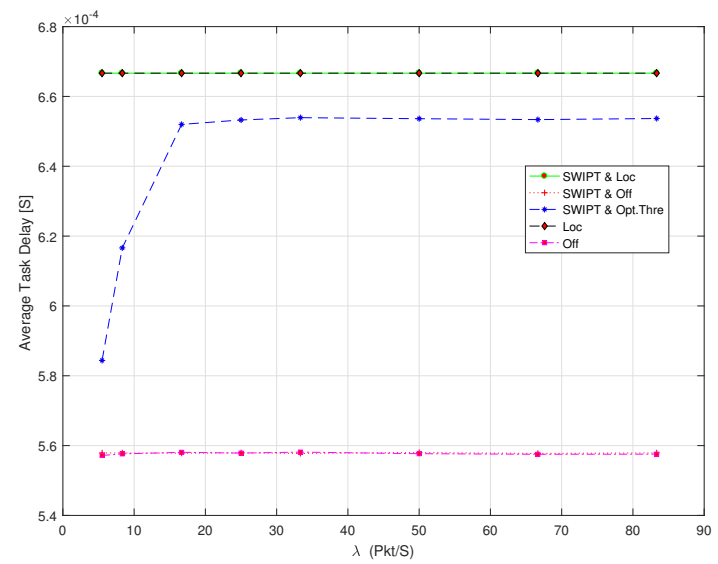

Fig. 5. Average Task Delay

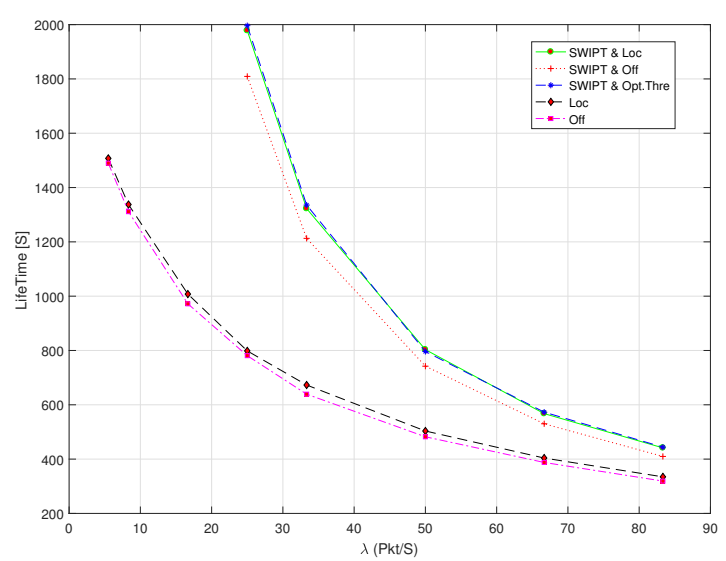

Fig. 6. Node Lifetime
SWIPT technology with always doing local processing and SWIPT technology when always offloading, respectively. Finally, Loc and Off consider the cases in which the FN is always performing local and always offloading without harvesting with SWIPT technology.

Fig. 5 depicts the average task delay for the 5 scenarios. As seen, the local scenarios are overlapping and having the highest delay due to the fact that packet size and computational power of an FN are fixed in this experiment. On the other hand, the offloading scenarios also overlap and have the lowest delay due to the higher computational power of the F-AP. However, the proposed solution, in which the decision is done based on the arrival of the next packet and the calculation of the two thresholds, lies in the middle of the other curves. As seen, when the $\lambda$ is small, it takes a longer time to have the next packet for computation, therefore there is a longer time for harvesting. As a result next packet arrival time is usually in area 3 which results in offloading decision to be made based on task delay. Therefore, because offloading takes a shorter time, the algorithm opts offloading. However, as $\lambda$ increases the arrival of next packet gets shorter and it lies mostly in area 2 (and sometimes in area 3 ) in which the lower threshold is selected. In the end, for packet generation rates higher than around $16 \mathrm{pkt} / \mathrm{s}$, the arrival time is always early, so that the FN opts the local computation and gets closer to the local computation scenarios.

Fig. 6 depicts the node lifetime for the 5 scenarios corresponding to the time instant the node goes off. As seen in the figure, scenarios exploiting SWIPT technology have a longer lifetime due to the energy harvesting, and the FN does not even go off when the $\lambda$ is small. As $\lambda$ increases more packets are generated for computation which results in more consumption, therefore, lifetime decreases for all scenarios. Moreover, the proposed solution has the longest lifetime.

As seen in Figs. 5 and 6, the proposed solution allows to select the offloading decision in a way that it benefit from both offloading and local computation. The proposed approach has a lower delay than the SWIPT \& local, where it benefits from the low delay of offloading, and a slightly higher lifetime with respect to the SWIPT \& local where it benefits from its lower energy consumption.

\section{Conclusions}

In this work, we have studied the impact of packet size and bandwidth on defining some thresholds which are later used for a computation offloading decision from an FN to an F-AP in a fog computing scenario. We have shown that exploiting SWIPT technology enables the FN to harvest energy and by estimating the arrival time of the following packet, considering the Poisson distribution, the $\mathrm{FN}$ is able to make the best offloading decision in order to shorten the task latency and extend the network lifetime. In the future, we will be investigating a multi-user and multi-F-AP scenario in which the problem is not only for offloading decision but also on the selection of devices for offloading.

\section{REFERENCES}

[1] L. M. Vaquero and L. Rodero-Merino, "Finding your way in the fog: Towards a comprehensive definition of fog computing," SIGCOMM Comput. Commun. Rev., vol. 44, no. 5, pp. 27-32, Oct. 2014.

[2] M. Xia and S. Aissa, "On the efficiency of far-field wireless power transfer," IEEE Trans. Signal Process., vol. 63, no. 11, pp. 2835-2847, Jun. 2015.

[3] L. R. Varshney, "Transporting information and energy simultaneously," in 2008 IEEE International Symposium on Information Theory, Toronto, ON, Canada, Jul. 2008, pp. 1612-1616.

[4] R. Ishikawa and K. Honjo, "High-efficiency DC-to-RF/RF-to-DC interconversion switching module at C-band," in 2015 European Microwave Conference (EuMC), Paris, France, Sep. 2015, pp. 295-298.

[5] T. D. P. Perera, D. N. K. Jayakody, S. K. Sharma, S. Chatzinotas, and J. Li, "Simultaneous wireless information and power transfer (SWIPT): Recent advances and future challenges," IEEE Commun. Surveys Tuts., vol. 20, no. 1, pp. 264-302, First Quarter 2018.

[6] F. K. Ojo and M. F. Mohd Salleh, "Throughput analysis of a hybridized power-time splitting based relaying protocol for wireless information and power transfer in cooperative networks," IEEE Access, vol. 6, pp. 24137 $24147,2018$.

[7] IEEE Standard for Safety Levels with Respect to Human Exposure to Radio Frequency Electromagnetic Fields, $3 \mathrm{kHz}$ to $300 \mathrm{GHz}$, IEEE Std. C95.1-2005, Apr. 2006. 